\title{
Cladosporium spp: Morfologia, infecções e espécies patogênicas
}

\author{
Camilla Pinheiro de Menezesa ${ }^{a^{*}}$, Ana Luíza Alves de Lima Péreza, Edeltrudes de Oliveira Limaab \\ ${ }^{a}$ Departamento de Ciências Farmacêuticas, Universidade Federal da Paraíba, João Pessoa, 58051-970, Brasil. \\ *camilla.farmaufpb@gmail.com

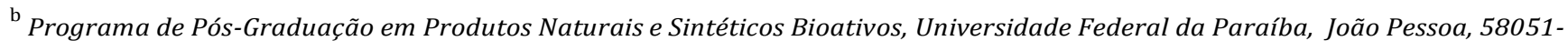 \\ 970, Brasil
}

Recebido: 12 dezembro 2016 / Aceito: 3 janeiro 2017 / Publicado online: 15 fevereiro 2017

\begin{abstract}
Resumo
O gênero Cladosporium spp compreende grande número de fungos dematiáceos com distribuição mundial e que estão entre os fungos de ambiente mais comuns. São frequentemente isolados como contaminantes, no entanto, algumas espécies são patogênicas e toxigênicas para os seres humanos, estando associados a infecções superficiais da pele e dos tecidos moles e incluem sepse disseminada com elevada mortalidade. Cladoporium spp são aero-alérgenos e causam graves doenças alérgicas do trato respiratório, bem como lesões intrabrônquicas. Atualmente, as espécies de Cladosporium spp de interesse médico associadas com doença humana são C. cladosporioides, C. herbarum, C. oxysporum e C. sphaerospermum. No caso de infecções por Cladosporium spp, a terapia é prolongada e varia de acordo com a síndrome clínica. Sendo essencial o conhecimento a cerca desse gênero, para melhor diagnóstico e tratamento adequado. Esta revisão de literatura teve como objetivo reunir informações sobre as características gerais e morfológicas, patologias, aspectos clínicos e principais espécies patogênicas das infecções causadas por esse gênero.
\end{abstract}

Palavras-chave: Feo-hifomicoses, Fungos dematiáceos, Micoses oportunistas.

\section{Cladosporium spp: Morphology, infections and pathogenic species}

\begin{abstract}
The genus Cladosporium spp comprises a large number of dematiaceous fungi with a world-wide distribution and are among the most common air-borne fungi. They are frequently isolated contaminants and some species are pathogenic and toxigenic to humans and are associated with superficial infections of the skin and soft tissues, including disseminated sepsis with high mortality. In addition, Cladoporium spp. are aero-allergens and cause serious allergic diseases of the respiratory tract, as well as intrabronchial lesions. Currently, the Cladosporium spp species of medical interest associated with human disease are $C$. cladosporioides, C. herbarum, C. oxysporum and C. sphaerospermum. In the case of Cladosporium spp infections, the therapy is prolonged and varies according to the clinical syndrome. Knowledge about this genre is essential for better diagnosis and appropriate treatment. This literature review to gather information about the general and morphological characteristics, pathologies, clinical aspects and main pathogenic species of the infections caused by this genus.
\end{abstract}

Keywords: Phaehyphomycoses, dematiaceus fungi, opportunistic mycoses.

\section{Introdução}

Os fungos fazem parte do ecossistema aerobiológico e apresentam-se em grandes quantidades, devido a sua grande capacidade de colonizar diferentes substratos e crescer em condições ambientais extremas. Alguns deles apresentam habilidade de causar doenças nos seres humanos, animais e vegetais (Gambale et al., 1993). Entre os vários táxons destaca-se Cladosporium spp, que é considerado um dos mais cosmopolitas e de maior concentração na atmosfera, particularmente em regiões temperadas (Zoppas et al., 2011).

O gênero Cladosporium spp, criado por Link, em 1816, é um dos maiores e mais heterogêneos gêneros de Hyphomycetes (Dugan et al., 2004). Este gênero compreende mais de 189 espécies, estando entre os fungos mais comumente isolados do ambiente em quase todo lugar do mundo, podendo ser isolado a partir de praticamente qualquer fonte ambiental e localização geográfica (Bensch et al., 2012; 2015).

Muitas espécies são conhecidas por serem patógenos de plantas, causando manchas foliares e outras lesões, ou parasitando outros fungos. As espécies de Cladosporium spp são também conhecidos por serem endófitos comuns (Revankar e Sutton, 2010).

São regularmente encontrados como contaminantes e agentes de deterioração nos alimentos ou produtos industriais, podendo ser isolados a partir de uma vasta gama de substratos, tais como terra, pedras, tijolos, bem como os têxteis, papel e couro, além de ser frequentemente associados com queixas 
asmáticas (Bensch et al., 2012; Gutarowska, 2014).

Conídios de espécies de Cladosporium spp representam o mais comum componente de fungos isolados do ar, uma vez que, são pequenos e bem adaptados para se espalhar facilmente em grandes números e por longas distâncias. São importantes alérgenos, e em grandes quantidades, podem afetar gravemente as pessoas asmáticas e com doenças respiratórias, pois, a exposição prolongada pode enfraquecer o sistema imunológico (Fairs et al., 2010).

Diante da facilidade de adaptação, contaminação e colonização dos diferentes ambientes e substratos torna-se essencial o conhecimento do gênero Cladosporium spp. Esta revisão de literatura teve como objetivo reunir informações sobre as características gerais e morfológicas, patologias, aspectos clínicos e principais espécies patogênicas das infecções causadas por esse gênero.

\section{Características Gerais e Morfológicas}

Os fungos pertencentes ao gênero Cladosporium spp são denominados fungos demácios, mielinizados ou pretos por apresentarem coloração naturalmente acastanhada em decorrência da presença de pigmento melânico (dihidroxinaftalenomelanina) em sua parede celular. Este pigmento, além de constituir um elemento fotoprotetor, é considerado um fator de virulência do fungo, uma vez que protege os organismos do estresse ambiental (exposição a metais pesados, dissecação, condições hiperosmóticas e temperaturas extremas), do antagonismo de outros organismos, da limitação de nutrientes, do choque de $\mathrm{pH}$ e contra a radiação ionizante e UV (Zaitz et al., 2012; Sidrim e Rocha, 2012).

$\mathrm{Na}$ fase saprófita, formam hifas septadas e escuras, com conidióforos laterais e terminais de tamanhos variados. A conidiação é do tipo Cladosporium spp, isto é, apresenta conidióforos de comprimento variado, eretos e variável quanto as suas ramificações, próximo ao ápice. Os conidióforos são de vários tamanhos e podem ser septados. Eles são pretos esverdeados claros e levemente dilatados nas extremidades distais. Produzem cadeias longas e ramificadas de conídios ovais e de paredes lisas e finas, à maneira de uma árvore, ou seja, as cadeias ramificam onde quer que um conídio produza dois brotos, ao em vez de um. Os blastoconídios podem apresentar cicatrizes escuras, nas disjunções ou nos hilos de onde se desgarram do conidióforo, ou de um outro conídio (Figura 1A; Fisher e Cook, 2001).

Os conídios podem ser produzidos em cadeias, sendo catenulados, muitas vezes solitários em algumas espécies, onde os conídios são mais largos e algumas vezes são ramificados em cadeia acropleurógena. Eles são simples, assumindo as formas cilíndricas, ovóides, doliformes, fusiformes, elipsóides, esféricas ou sub-esféricas. Possuem coloração marrom olivácea escuro ou marrom e a superfície do conídio pode ser lisa, verrugosa ou equinulada com 0 a 3 septos, ocasionalmente (Figura 1B; Bensch et al., 2012).

A incapacidade das espécies de Cladosporium spp para crescer em meios contendo cicloheximida, presença de células shield, ou hilo proeminente (cicatrizes de fixação) e conídios frágeis que se desprendem facilmente são características que distinguem as espécies de Cladosporium spp das espécies de Cladophialophora (Revankar e Sutton, 2010).

Nos tecidos aparecem como células leveduriformes, pseudo-hifas, hifas verdadeiras ou qualquer combinação dessas formas, com coloração castanha (Zaitz et al., 2012).

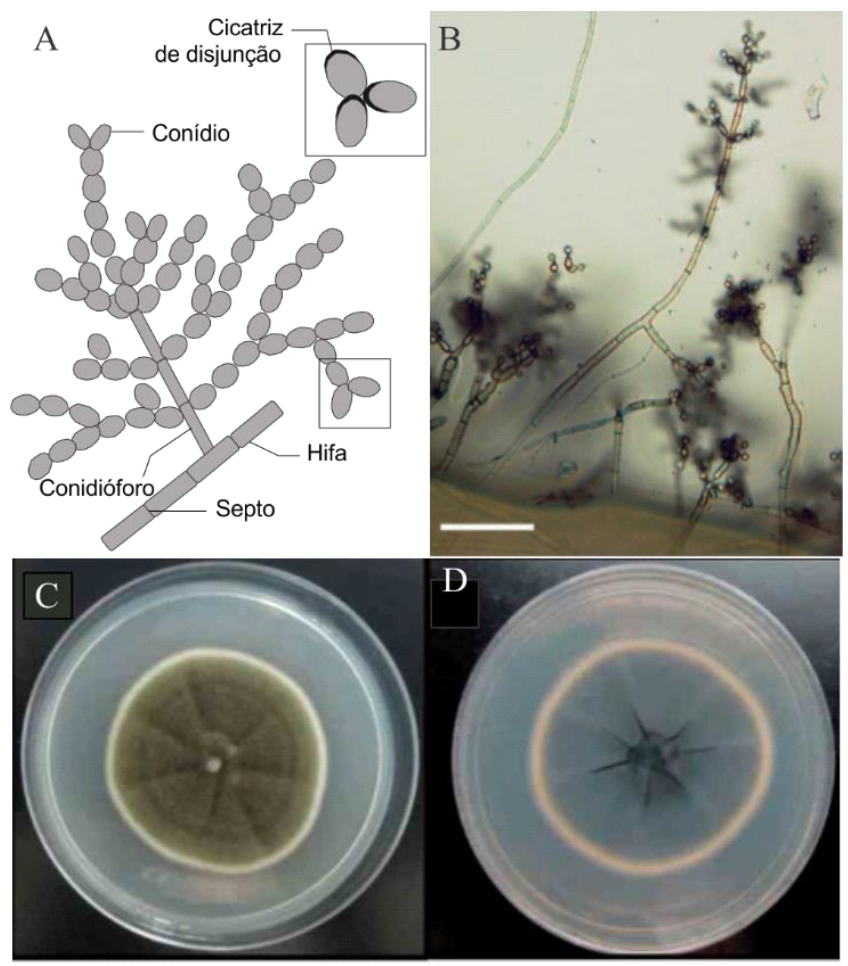

Figura 1. Características morfológicas de Cladosporium spp. A: Conidiação do tipo Cladosporium spp (Adaptado Fisher e Cook, 2001). B: Micromorfologia. Barra de escala: $50 \mu \mathrm{m}$ (Adaptado de Zalar et al., 2007). C e D: Macromorfologia, verso e reverso da colônia após 14 dias de crescimento em ágar sabouraund dextrose a $25^{\circ} \mathrm{C}$ (Adaptado de Shi et al., 2016).

São fungos de crescimento lento, atingindo a maturidade dentro de 14 a 21 dias. Caracterizam-se pela produção de colônias efusas ou ocasionalmente puntiformes, com superfícies planas, aveludadas, circulares, de crescimento lento e enrugado, que vão do verde oliva ao marrom escuro e reverso preto (Figura $1 \mathrm{C}$ e D; Tamsikar et al., 2006).

\section{Patogênese e manifestações clínicas}

Os fungos dematiáceos, como os pertencentes ao gênero Cladosporium spp, têm a propriedade de digerir as proteínas da epiderme, causando várias lesões de pele, desde pequenas manchas avermelhadas até severas erupções que podem abrir caminho para a disseminação desses fungos, causando a proliferação das lesões, podendo comprometer parcial ou totalmente alguns órgãos (Espinel-Ingroff et al., 1986).

$\mathrm{O}$ espectro de doenças com as quais estão associados incluem desde infecções superficiais de pele e tecidos moles até sepse disseminada, com elevada mortalidade (Yew et al., 2014). As infecções mais comuns incluem feo-hifomicoses, doença alérgica, infecções superficiais e profundas locais, pneumonia, abscesso cerebral, e infecção disseminada. Para 
algumas infecções em indivíduos imunocompetentes, tais como sinusite fúngica alérgica e abscesso cerebral, eles estão entre os agentes etiológicos mais comuns (Zaitz et al., 2012; Revankar e Sutton, 2010).

As feo-hifomicoses, de acordo com McGinnis (1983), englobam importante, distinto e heterogêneo grupo de infecções micóticas causadas por fungos dematiáceos que vão desde o comprometimento superficial até doenças em órgãos profundos, nas quais of $\mathrm{s}$ agentes etiológicos ocorrem nos tecidos como células leveduriformes, pseudo-hifas e hifas demacioides curtas ou alongadas, regulares, distorcidas ou dilatadas ou em combinação com quaisquer destas formas (Sidrim e Rocha, 2012).

Os achados clínicos das feo-hifomicoses são bastante variáveis, dependendo do padrão imunológico do hospedeiro, sítio anatômico acometido e espécie fúngica implicada, podendo assim ser classificada como superficial, subcutânea e sistêmica ou invasivas. As formas invasivas mais comuns são as pulmonares e cerebrais (Castro et al., 2013; Singh et al., 2005).

As micoses superficiais são infecções subagudas ou crônicas da camada superficial da pele, pelos e unhas. Caracterizam-se por provocar quadros clínicos confinados ao extrato córneo, de forma análoga à dermatomicose, ceratite micótica e onicomicoses (Schwartz, 2004; Trabulsi e Alterthum, 2016).

A micose subcutânea é a forma clínica mais comum de feo-hifomicose, caracterizando-se por um quadro infeccioso de evolução crônica, que tem como origem um traumatismo cutâneo. No local do traumatismo, ocorre a inoculação do fungo, que evolui com formação de nódulos contendo secreção sanguinolenta ou seropurulenta (Sidrim e Rocha, 2012; Trabulsi e Alterthum, 2016).

A micose sistêmica é a forma clínica mais rara, sendo encontrada, sobretudo, em pacientes imunossuprimidos (diabéticos, leucêmicos, pacientes debilitados, com doenças crônicas e por uso de drogas imunossupressoras). As feohifomicoses sistêmicas acometem por excelência os órgãos internos, especialmente o cérebro. No entanto, outros órgãos podem ser afetados, como por exemplo, coração, intestino, ossos, baço, fígado e rins. Acredita-se que a principal forma de infecção é a inalação (Singh et al., 2005; Sidrim e Rocha, 2012).

Os conídios de Cladosporium spp têm sido associados à rinite e asma alérgica (Vicendese et al., 2015). As doenças alérgicas, bem como a sinusite fúngica causadas por Cladosporium spp, podem ocorrer de forma não invasiva que acomete indivíduos atópicos, imunocompetentes. Está, na maioria das vezes, associada à polipose nasal e à asma, tendo como característica principal a presença de mucina alérgica. Ou de forma crônica e invasiva que ocorre em indivíduos imunocompetentes, não atópicos, com história de rinossinusite crônica, podendo levar a alterações na função pulmonar (Boechat et al., 1998; Chen et al., 2014).

Os sintomas variam de drenagem pós-nasal purulenta a obstrução nasal progressiva grave, com eliminação de massa de coloração marrom pelas narinas. Podem estar presentes distúrbios visuais, por compressão do globo ocular ou nervo óptico, dor facial e sudorese. Em jovens, nos quais as estruturas sinusais não estão completamente calcificadas, a infecção fúngica pode levar à proptose, ao hipertelorismo ou à deformidade malar (Boechat et al., 1998).

\section{Principais espécies patogênicas}

Apesar de sua prevalência, apenas um número limitado de espécies têm sido documentados como agentes de infecções micóticas em humanos. As espécies bem conhecidas como patogênicas em humanos, anteriormente conhecidas como Cladosporium bantiana, Cladosporium carrionii e Cladosporium devriesii, caracterizada pela ausência de conidióforos, e cicatrizes conidiais não pigmentadas, foram reclassificadas no gênero Cladophialophora (Bensch et al., 2012). De modo que, atualmente, as espécies de Cladosporium spp de interesse médico, associadas com doença humana são Cladosporium cladosporioides, Cladosporium herbarum, Cladosporium oxysporum e Cladosporium sphaerospermum (De Hoog et al., 2011).

Estas espécies geralmente estão associadas à rinite e asma alérgica (Chen et al., 2014; Vicendese et al., 2015), quadro de feo-hifomicoses superficiais, feo-hifomicoses profundas (Gugnani et al., 2006; Martinez-Herrera et al., 2015), e mas raramente podem causar infecções disseminadas (SandovalDenis et al., 2015).

C. sphaerospermum é uma espécie saprofítica, cosmopolita que habita uma variedade de ambientes. É encontrada ubiquamente em ambientes naturais e antropizados, tais como: $\mathrm{o}$ ar interior e o exterior, o solo, a vegetação em decomposição, pintura, silicone e têxteis, podendo também está presente nas plantas, animais e seres humanos. É um importante fitopatógeno, sendo muito prejudicial para as culturas vegetais (Zalar et al., 2007). Para os seres humanos e animais, nem todas as cepas são patogênicas, entretanto algumas cepas podem provocar ocasionalmente feo-hifomicose cutânea e cerebral independentemente do estado imunitário do hospedeiro (Soumagne et al., 2015; Maduri et al., 2015).

C. cladosporioides é um fungo, dematiáceo, saprofítico amplamente distribuído nos mais variados ambientes que ocasionalmente pode provocar uma variedade de infecções clínicas em seres humanos e animais imunocomprometidos e imunocompetentes. As manifestações clínicas variam dependendo da imunidade do hospedeiro e dos tecidos atingidos (Shi et al., 2016).

C. oxysporum é um fungo saprofítico que frequentemente cresce em vários substratos. Esta espécie é muitas vezes encontrada como contaminantes da culturas vegetais (Zheng et al., 2014) e citada em casos de doença cutânea e subcutânea (Romano et al., 1999; Gugnani et al., 2006).

C. herbarum é amplamente distribuído no ambiente e é uma importante fonte de alérgenos fúngicos inalantes. É um dos fungos ambientais mais comuns, podendo ser isolado em todo o mundo. Essa espécie ocorre abundantemente em folhas mortas de plantas herbáceas e lenhosas, como um invasor secundário em manchas foliares necróticas, e tem sido frequentemente isolado do ar, alimentos, tintas, têxteis, os seres humanos e vários outros substratos (Schubert et al., 2007). Nos seres humanos, está relacionado com quadros clínicos de alergias e intoxicações (Pandey et al., 2016). 


\section{Tratamento}

Em se tratando de infecções por fungos dematiáceos a terapia é prolongada e varia de acordo com a síndrome clínica. Infecção local pode ser curada com excisão isolada, enquanto a doença sistêmica é muitas vezes refratária ao tratamento, sendo necessária, em geral a combinação de terapias (Revankar e Sutton, 2010).

A diversidade de quadros de feo-hifomicose impõe condutas particulares, de modo que o tratamento das feohifomicoses é bastante variável no que se refere ao tipo, localização e à extensão da lesão, bem como ao fungo envolvido e ao estado imunitário do paciente, dependendo, portanto da forma clínica (Revankar e Sutton, 2010).

$\mathrm{O}$ tratamento de primeira escolha para as feo-hifomicoses é a terapia sistêmica oral com um antifúngico da classe do azólicos, sendo o fármaco de escolha o itraconazol em esquema prolongado (Zhou et al., 2016). A terbinafina, antifúngico pertencente a classe das alilaminas também tem sido utilizada com sucesso, particularmente em pacientes que não responderam a terapia com azólicos (Agger et al., 2004).

Em casos de infecções mais graves pode-se associar a anfotericina B e o voriconazol (Chen et al., 2014). Lesões localizadas devem ser tratadas cirurgicamente ou com infiltrações locais de anfotericina B e para casos disseminados emprega-se a associação de anfotericina B e 5fluorocitosina (Deng et al., 2016).

Nos casos alérgicos ou de sinusite fúngica, o tratamento consiste em procedimentos cirúrgicos e medicamentosos. Os procedimentos cirúrgicos para remoção da mucina alérgica e restos celulares necróticos têm função diagnóstica e terapêutica. A cirurgia proporciona alívio imediato dos sintomas obstrutivos, melhorando a drenagem das secreções e reduzindo a carga fúngica local. Entretanto é difícil a eliminação completa dos fungos (Boechat et al., 1998).

Os corticosteróides sistêmicos são considerados a medicação de escolha no pós-operatório da sinusite fúngica. Atuam diminuindo a resposta inflamatória, reduzindo o edema de mucosa, a secreção e a formação de pólipos (Roth, 1994). Antifúngicos sistêmicos têm sido ineficazes na sinusite fúngica, devido à localização extramucosa do fungo, estando, portanto, fora do alcance da droga circulante. Para que produza efeito, um antifúngico sistêmico deve ser secretado no muco sinusal, o que não tem sido documentado. Irrigações locais com antifúngicos, principalmente, cetoconazol e anfotericina $\mathrm{B}$, iniciadas no período préoperatório e se estendendo por vários meses após a cirurgia, diminuem a recolonização fúngica (Bent e Kuhn, 1996).

\section{Referências}

Agger, W.A.; Andes, D.; Burgess, J.W. 2004. Exophiala jeanselmei infection in a heart transplant recipient successfully treated with oral terbinafine. Clinical Infectious Disease, 38: 112-115.

Bensch, K.; Braun, U.; Groenewald, J.Z.; Crous, P.W. 2012. The genus Cladosporium. Studies in Mycology, 72: 1-401.

Bensch, K.; Groenewald, J.Z.; Braun, U.; Dijksterhuis, J.; Yañes-Morales, M.J.; Crous, P.W. 2015. Common but different: the expanding realm of Cladosporium. Studies in Mycology, 82: 23-74.

Bent, J.P; Kuhn, F.A. 1996. Antifungal activity against allergic fungal sinusitis organisms. Laryngoscope, 106(11): 1331-1334.

Boechat, J.L.; De La Reza, D.; Abe, A.T.; Valle, S.O.R.; França, A.T. 1998.
Sinusite fúngica alérgica: atualização. Revista Brasileira de alergia e imunopatologia, 21(4): 105-111.

Castro, A.S.; Oliveira, A.; Lopes, V. 2013. Pulmonary phaeohyphomycosis: a challenge to the clinician. European Respiratory Review, 22(128): 187192.

Chen, B.Y.; Chao, H.J.; Wu, C.F.; Honda, Y.; Guo, Y.L. 2014. High ambient Cladosporium spores were associated with reduced lung function in schoolchildren in a longitudinal study. Science of the Total Environment, 481(2014): 370-376.

De Hoog, G.S.; Guarro, J.; Gené, J.; Filgueiras, M.J.2011. Atlas of Clinical Fungii. CD-ROM versão 3.1. CBS-KNAW, Utrecht, Holanda.

Deng, S.; Pan, W.; Liao, W.; De Hoog, G.S.; Gerrits Van Den Ende, A. H G.; Vitale, R.G.; Rafati, H.; Ilkit, M.; Van Der Lee, A.H.; Rijs, A.J.M. M.; Verweij, P.E.; Seyedmousavi, S. 2016. Combination of amphotericin B and flucytosine against neurotropic species of melanized fungi causing primary cerebral phaeohyphomycosis. Antimicrobial Agents Chemotherapy, 60(4): 2346-2351.

Dugan, F.M.; Schubert, K.; Braun, U. 2004. Check-list of Cladosporium names. Schlechtendalia, 11: 1-103.

Espinel-Ingroff, A.; Shadomy, S.; Dixon, D.; Goldson, P. 1986. Exoantigen test for Cladosporium bantianum, Fonsecaea pedrosoi and Phialophora verrucosa. Journal of Clinical Microbiology, 23(2): 305-310.

Fairs, A.; Agbetile, J.; Hargadon, B.; Bourne, M.; Monteiro, W.R.; Brightling, C.E.; Bradding, P.; Verde, R.H.; Mutalithas, K.; Desai, D.; Pavord, I.D.; Wardlaw, A.J.; Pashley, C.H. 2010. IgE sensitization to Aspergillus fumigatus is associated with reduced lung function in asthma. American Journal of Respiratory and Critical Care Medicine, 182(11): 1362-1368.

Fisher, F.; Cook, N.B. 2001. Micologia Fundamentos e Diagnóstico. 1. ed. Rio de Janeiro: Revinter. 337 p.

Gambale, W.; Croce, J.; Costa-Manso, E.; Croce, M.; Sales, M. 1993. Library fungi at the University of São Paulo and their relationship with respiratory allergy. Journal of Investigational Allergology and Clinical Immunology, 3(1): 45-50.

Gutarowska, B. 2014. Moulds in biodeterioration of technical materials. Folia Biologica et Oecologica, 10(1): 27-39.

Gugnani, H.C.; Ramesh, V.; Sood, N.; Guarro, J.; Moin-Ul-Haq; PaliwalJoshi, A.; Singh, B. 2006. Cutaneous phaeohyphomycosis caused by Cladosporium oxysporum and its treatment with potassium iodide. Medical Mycology, 44(3): 285-288.

Maduri, A.; Patnayak, R.; Verma, A.; Mudgeti, N.; Kalawat, U.; Asha, T. 2015. Subcutaneous infection by Cladosporium sphaerospermum - A rare case report. Indian Journal of Pathology and Microbiology, 58(3): 406-407.

Martinez-Herrera, E.O.; Arroyo-Camarena, S.; Tajada-Garcia, D. L.; PorrasLopez, F. Arenas, R. 2015. Onychomycosis due to opportunistic molds. Anais Brasileiro de Dermatologia, 90(3): 334-337.

McGinnis, M.R. 1983. Chromoblastomycosis and phaeohyphomycosis: new concepts, diagnosis, and mycology. Journal of The American Academic Dermatology, 8(1): 1-16.

Pandey, S.P.; Fuhrman, C.; Bittar, H.E.T. 2016. The first reported case of severe organic dust toxicity syndrome caused by Cladosporium herbarum. American Journal of Respiratory and Critical Care Medicine, 193: A7948.

Revankar, S.G.; Sutton, D.A. 2010. Melanized fungi in human disease. Clinical Microbiology Reviews, 23(4): 884-928.

Romano, C.; Bilenchi, R.; Alessandrini, C.; Miracco, C. 1999. Case Report. Cutaneous phaeohyphomycosis caused by Cladosporium oxysporum. Mycosis, 42(1-2): 111-115.

Roth, M. 1994. Should oral steroids be the primary treatment for allergic fungal sinusitis? Ear Nose Throat Journal, 73(12): 928-930.

Sandoval-Denis, M.; Sutton, D.A.; Martin-Vicente, A.; Cano-Lira, J.F.; Wiederhold, N.; Guarro, J.; Gené, J. 2015. Cladosporium species recovered from clinical samples in the United States. Journal of Clinical Microbiology, 53(9): 2990-3000.

Schubert, K.; Groenewald, J.Z.; Braun, U.; Dijksterhuis, J.; Starink, M.; Hill, C.F.; Zalar, P.; De Hoog, G.S.; Crous, P.W. 2007. Biodiversity in the Cladosporium herbarum complex (Davidiellaceae, Capnodiales), with standardisation of methods for Cladosporium taxonomy and diagnostics. Studies in Mycology, 58: 105-156.

Schwartz, R.A. 2004. Superficial fungal infections. Lancet, 364(9440): $1173-$ 1182. 
Shi, D.; Lu, G.; Mei, H.; Shen, Y.; Qiu, Y.; Liu, W. 2016. A rare case of onychomycosis induced by Cladosporium cladosporioides. Journal of Clinical and Medicine Case Reports, 2(2): 1072.

Sidrim, J.J.C.; Rocha, M.F.G. 2012. Micologia médica à luz de autores contemporâneos [Reimpressão]. Rio de Janeiro: Guanabara Koogan. $372 \mathrm{p}$.

Singh, S.; Singh, P. Sarkar, C.; Goel, V.; Srivastava, T., Sharma, M. C.; Behari, M. 2005. Fungal granuloma of the brain caused by Cladosporium bantianum - a case report and review of literature. Journal of the Neurological Sciences, 228(1): 109-112.

Soumagne, T.; Pana-Katatali, H.; Degano, B.; Dalphin, J.C. 2015. Combined pulmonary fibrosis and emphysema in hypersensitivity pneumonitis. BMJ Case Reports.

Tamsikar, J.; Naidu, J.; Singh, S.M. 2006. Phaeohyphomycotic sebaceous cyst due to Cladosporium cladosporidioides: case report and review of literature. Journal of Medical Mycology, 16(1): 55-57.

Tasic, S.; Tasic, N.M. 2007. Cladosporium spp.- cause of oportunistic mycoses. Acta Facultatis Medicae Naissensis, 24(1): 15-19.

Trabulsi, L.R.; Alterthum, F. 2016. Microbiologia. 6. ed. São Paulo: Atheneu. 920p.

Vicendese, D.; Dharmage, S.C.; Tang, M.L.; Olenko, A.; Allen, K.J.; Abramson, M.J.; Erbas, B. 2015. Bedroom air quality and vacuuming frequency are associated with repeat child asthma hospital admissions. Journal Asthma: Official Journal of the Association for the Care of Asthma, 52(7): 727-31.

Yew, S.M.; Chan, C.L.; Lee, K.W.; Na, S.L.; Tan, R.; Hoh, C.C.; Yee, W.Y.; Ngeow, Y.F.; Ng, K.P. 2014. A five-year survey of dematiaceous fungi in a tropical hospital reveals potential opportunistic species. PLoS One, 9(8): e104352.

Zaitz, C.; Campbell, I.; Marques, S.A.; Ruiz, L.R.B.; Framil, V.M.S. 2012. Compêndio de micologia médica. 2. ed. Rio de Janeiro: Guanabara Koogan. 432p.

Zalar, P.; De Hoog, G.; Schroers, H.; Crous, P.; Groenewald, J.; GundeCimerman, N. 2007. Phylogeny and ecology of the ubiquitous saprobe Cladosporium sphaerospermum, with descriptions of seven new species from hypersaline environments. Studies in Mycology, 58: 157-183.

Zheng, C.; Liu, Z.H.; Tang, S.S.; Lu, D.; Huang, X.Y. 2014. first report of leaf spot caused by Cladosporium oxysporum on greenhouse eggplant in China. Plant Disease, 98(4): 566-566.

Zhou, Y.B.; Chen, P.; Sun, T.T.; Wang, X.J.; Li, D.M. 2016. Acne-like subcutaneous phaeohyphomycosis caused by Cladosporium cladosporioides: a rare case report and review of published literatures. Mycopathologia, 181 (7-8): 567-573.

Zoppas, B.C.A.; Valencia-Barrera, R.M.; Fernandéz-Gonzáles, D. 2011 Distribuição de esporos de Cladosporium spp no ar atmosférico de Caxias do Sul, RS, Brasil, durante dois anos de estudo. Revista Brasileira de Alergia e Imunopatologia, 34(2): 55-58. 